\title{
13. DISCUSSION FOLLOWING SPIEGEL'S REPORT
}

(Thursday, September 18, 1969)

Chairman: I. S. SHKLovSKII

[The text of this Chapter contains the first part of the Discussion on Thursday, September 18, 1969; the second part is given in Chapter 26. I added a long contribution on cosmogonical problems, presented by Dr. Ozernoi on September 10.]

Weymann: Dr. Spiegel, did you assume a (more or less) uniform distribution of matter throughout the Coma-cluster?

Spiegel: Yes. In the theory I have, one does the hydrodynamics as if the gas were adiabatic and then one calculates the radiation from the resulting conditions. So far I do not have a theory which includes radiation ab initio.

Weymann: What I worry about is whether or not the gas is condensed towards the center. Since the cooling rate is proportional to $n^{2}$ ( $n$ is the density) it is conceivable to me that an almost steady state is possible in which the gas in the center of the cluster is much cooler and much denser.

Spiegel: Quite so.

Ozernoi: In his exciting Report, Spiegel mentioned the interaction between galaxies and intergalactic gas. The importance of such interactions for the energy balance of radio galaxies was first suggested, as far as I know, by Shklovskii. I should like to discuss a recent paper (Ozernoi, 1969) on mass accretion of quasars and other systems. Consider Hubble's famous 'tuning-fork' diagram, which shows the morphological sequence of galaxies from $\mathrm{E}$ to Irr, including the branching into barred and nonbarred spirals. I extended this fork by adding quasars and similar sources and got a sequence QSS-N-BCG-D-E-S0-Sa, etc. [BCG stands for blue compact galaxy. (Ed.).] In this sequence, from right to left, both the radio and the optical luminosities increase; the specific angular momentum decreases. I think that the source of energy [in the series QSS through D? (Ed.)] is an object in the nuclei of such galaxies, a magnetoid, i.e., a very massive magneto-dynamic plasma configuration rotating in a magnetic field, analogous to a giant pulsar. The magnetodynamic model can explain the physics of quasars in much detail. However, the question remains, what final reservoir supplies the central parts of galaxies with the required mass of gas? A first possible reservoir is the non-fragmented gas in the galaxies themselves. Between two active phases of the nucleus, cooling of the gas and condensation toward the center, may supply gas to the nucleus. A second possible reservoir is accretion of an intergalactic gas cloud by a galaxy. The rate of accretion depends on the relation between the velocity of a galaxy $\mathrm{V}$ and the parabolic velocity on its border $V_{p} \propto(M / R)^{1 / 2}$. For a galaxy of given morphological type the mean density $\left\langle\varrho_{g}\right\rangle=$ const, i.e., $R \propto M^{1 / 3}$, and the radius of accretion $R_{A} \propto M$. Consequently, for galaxies of small masses, $R>R_{A}$ and the rate of accretion 
$\mathrm{d} M / \mathrm{d} t \propto M^{2 / 3} V$; meanwhile for most massive galaxies $R<R_{A}$, and $\mathrm{d} M / \mathrm{d} t \propto M^{2} V^{-3}$. The parameters $M, R, V$, which characterize a galaxy, cannot be chosen freely but are determined by the cosmological past of a galaxy. The analysis shows that in all real situations $\mathrm{d} M / \mathrm{d} t \propto M^{\alpha}$ with $\alpha>0$. Because the mean mass of a galaxy increases from Irr to E galaxies and, possibly, along all the extended sequence (from right to left), the rate of accretion would increase in the same direction. The accretion rate also depends on whether or not a galaxy is a member of a cluster. Field galaxies have velocity dispersions smaller by a factor of about 10 than galaxies in rich clusters. As a result the rate of accretion for galaxies of small masses would be greater inside clusters; meanwhile for most massive galaxies the rate of accretion would be greater outside clusters. This explains, possibly, the primary appearance of quasars and quasarlike phenomena outside clusters. After the gas has been captured by a galaxy, the rate at which it condenses into the center depends on the specific angular momentum of the galaxy. One expects that the rate of condensation would be greater, the smaller the specific angular momentum. Because the latter increases along the sequence from left to right, the luminosity of nonthermal emission (which is connected with transformed kinetic energy) would decrease in the same direction. The pressure of both radiation and relativistic particles as well as the outflow of the plasma from the nucleus restrict the inflow of metagalactic gas. Its condensation would repeat between active phases of the nucleus and, in principle, can explain the recurrent character of phenomena in quasars and the nuclei of galaxies. (Ozernoi, L. M.: 1969, Astron. Tsirk. No. 469.)

[The following part of the discussion took place on September 10.]

\section{Ozernoi: The Traces of 'Photon Whirls'.}

It is generally assumed that the observed inhomogeneities of the Universe (galaxies and galaxy clusters) originated as a result of gravitational instability of initially homogeneous matter with small, but finite (non-fluctuating) density perturbations. I should like, however, to discuss a different assumption, namely that the initial structure was not static, but dynamic: I assume that initially whirl (vortex) motions, both of the photon gas and the plasma, are superimposed on the cosmical expansion. I assume that vortex velocities are subsonic and (on sufficiently large scales) lead to a Kolmogorov spectrum, which is independent of the initial turbulence spectrum. The interval of $k$ (the wave number) over which the Kolmogorov spectrum holds is determined at the lower side by the condition that the hydrodynamic time equals the cosmological time. When in the expansion the moment comes that the metagalactic plasma recombines, the sound velocity becomes significantly lower and the (originally subsonic) whirls are suddenly in the supersonic regime. This leads to motions and inhomogeneities in the distribution of matter, even if originally this distribution was uniform. Depending on whether the inhomogeneities are gravitationally bound or not, predictions can be made for (i) the future velocity spectrum, (ii) the possibility that internal motions will remain smaller than the differential velocity of cosmological expansion, (iii) the spectrum of the density inhomogeneities, and (iv) the density contrast between 
the inhomogeneities and the background. Comparing our predictions with observations of groups and clusters of galaxies (Karantchentsev, 1966), we conclude that there is a fair agreement between predictions and observations, provided that clusters of galaxies represent, on the average, gravitationally bound systems, except on the size scale of superclusters. The fact that there is agreement supports the basic assumption of the existence of primeval cosmological turbulence. More details may be found elsewhere (Ozernoi and Chernin, 1967, 1968). [Comment condensed. (Ed.).] [Karantchentsev, I. D.: 1966, Astrofiz. 2, 81 (translation: 1966, Astrophys. 2, 39); Ozernoi, L. M. and Chernin, A. D.: 1967, Astron. Zh. 44, 1131 (translation: 1968, Soviet Astron. 11, 907); 1968, Astron. Zh. 45, 1137 (translation: 1969, Soviet Astron. 12, 901).]

Spiegel: I do not understand why you would expect vortex motions. In an inviscid fluid the Hamiltonian density contains both longitudinal and rotational terms. Is it not true that, as in a superfluid, the lowest excitations are the longitudinal ones?

Zel'dovich: The longitudinal quanta will be smaller than the rotational quanta. So in thermodynamic equilibrium you will have phonons but not rotons. But in our primeval Universe there is no thermodynamic equilibrium. It is simply assumed that at the origin you have vortices and no strong sound wave motions. Ozernoi is not bound to a situation of thermal equilibrium at the beginning of the Universe. Therefore, your remark, Dr. Spiegel, seems to be irrelevant. There are other difficulties with Ozernoi's theory. I think that at the moment of chemical element formation it is incompatible with the Friedman model. Perhaps the theory could be made compatible by assuming many neutrinos.

Van de Hulst: Oort has recently also been interested in the formation of galaxies; and, like you, he traces it back to gas-dynamic motions in an earlier epoch. He told me that he was much impressed by the fact that in many cases the galaxies seem to form strings. Do you think that this structure is important? Does it fit into your theory?

Ozernoi: It seems to me that such clustering of galaxies is connected with the details of space correlation in the primeval whirl structure. According to the whirl model this correlation is the main reason for the formation of the clusters of galaxies. It explains some important features of the clusters, for instance, the relationship between the mean density of a cluster and the predominant morphological type of its galaxies.

Colgate: Dr. Ozernoi, can you suggest what could have caused the turbulence in the first place?

Zel'dovich: Dr. Colgate, I should like to ask you: from where came our Universe? 